\title{
Reliability Analysis of Weighted- $k$-out-of- $n$ : G System Consisting of Two Different Types of Nonidentical Components Each with its Own Positive Integer-Valued Weight
}

\author{
Eisa Mahmoudi*(D), RahmatSadat Meshkat \\ Department of Statistics, Yazd University, 89175-741, Yazd, Iran
}

\section{ARTICLE INFO}

Article History

Received 5 May 2019

Accepted 14 June 2020

Keywords

Component importance

Mean time to failure

Reliability

Weighted- $k$-out-of- $n: \mathrm{G}$ system

2000 Mathematics Subject Classification: 62N05, 90B25, $60 \mathrm{~K} 10$

\begin{abstract}
This paper introduces a special case of weighted- $k$-out-of- $n: G$ system formed from two types of nonidentical components with different weights. This system consists of $n$ nonidentical components each with its own positive integer-valued weight which are categorized into two groups with respect to their duties and services. In fact, we have a system consisting $n$ components such that $n_{1}$ of them each with its own weight $\omega_{i}$ and reliability $p_{1 i}$ and $n_{2}$ of them each with its own weight $\omega_{i}^{*}$ and reliability $p_{2 i}$. If the total weights of the functioning components exceeds a prespecified threshold $k$, the system is supposed to work. The reliability of system is obtained based on the total weight of all working components in both group. The survival function and mean time to failure are presented. Also, the component importance of this system are studied.
\end{abstract}

(C) 2020 The Authors. Published by Atlantis Press B.V. This is an open access article distributed under the CC BY-NC 4.0 license (http://creativecommons.org/licenses/by-nc/4.0/).

\section{INTRODUCTION}

In most real-life systems, the total contribution of the components plays an important role and must be above a specified performance level. In many situations, the components contribute differently to the capacity of the system. The systems with weighted components with unequal weights are introduced by $\mathrm{Wu}$ and Chen [1] to deal with this situation which has been studied in the literature. A system including $n$ components with their different positive integer weights is known as weighted- $k$-out-of- $n$ :G system when it works if and only if the total weight of working components is above a given threshold $k$.

Chen and Yang [2] extended the one-stage weighted- $k$-out-of- $n$ model to the two-stage weighted- $k$-out-of- $n$ model with components in common. Samaniego and Shaked [3] presented a review on weighted- $k$-out-of- $n$ systems. Li and Zuo [4] provided two models of multistate weighted- $k$-out-of- $n$ system models and presented the recursive algorithms for their reliability evaluation. Navarro et al. [5] extended the signature-based representations of the reliability functions of coherent systems to systems with heterogeneous components. Eryilmaz [6] introduced a $k$-out-of- $n$ system with random weights for components and investigated the reliability properties of the system. Eryilmaz and Bozbulut [7] studied a multi-state weighted- $k$-out-of- $n$ :G system model in a dynamic setup and yielded an algorithmic approach for its dynamic reliability analysis. In order to measure the components importance in $k$-out-of- $n$ system with random weights, Rahmani et al. [8] defined the weighted importance (WI) measure that depends only on the distribution of component weights and also, Meshkat and Mahmoudi [9] generalized this measure for two component $i$ and $j$ and the relation of these measures is investigated with Birnbaum reliability importance measure.

Eryilmaz and Sarikaya [10] studied the special case of weighted- $k$-out-of- $n$ : G system containing two types of components, each group having different weights and reliabilities such that one group have the common weight $\omega$ and reliability $p_{1}$, while the other have common weight $\omega^{*}$ and reliability $p_{2}$. They also obtained the nonrecursive equations for the system reliability, survival function and mean time to failure (MTTF). Recently, Eryilmaz [11] introduced the $\left(k_{1}, k_{2}, \cdots, k_{m}\right)$-out-of- $n$ system including $n_{i}$ components of type $i$ for $i=1, \cdots, m$ and $n=\sum_{i=1}^{m} n_{i}$. The corresponding system is assumed to work if at least $k_{1}$ components of type $1, k_{2}$ components of type $2, \ldots, k_{m}$ components of type $m$ function. Its reliability and the setup of weighted- $\left(k_{1}, k_{2}, \cdots, k_{m}\right)$-out-of- $n$ system is also defined and studied.

*Corresponding author. Email: emahmoudi@yazd.ac.ir 
In this study, we consider a general setup of weighted- $k$-out-of- $n: G$ system in which one group consists of $n_{1}$ components each with its own positive integer-valued weight $\omega_{i}$ and reliability $p_{1 i}$, while the other group consists of $n_{2}$ components each with its own positive integervalued weight $\omega_{i}^{*}$ and reliability $p_{2 i},\left(n=n_{1}+n_{2}\right)$. This system operates if the total weight of all working components is at least $k$. Therefore, its reliability is obtained based on the total weight of all working components in both group. This setup of weighted- $k$-out-of- $n$ :G system containing two types of nonidentical components might be useful in practice. As an illustration, assume that a production line (system) is constituted by $n$ devices (components) in two groups with $n_{1}$ and $n_{2}$ devices $\left(n=n_{1}+n_{2}\right)$, respectively, which each group has their own duties and services and different capacities (weights). For an instance, a production line in tile factory consists of two groups of devices, one group including some devices to combine the materials and bake the tiles and the other group including some devices to implement the layout and color on the tiles and glaze them. Finally to have a complete tile, some devices in the line should be operate such that a certain capacity of total capacities is provided. Therefore, we have a system consisting $n$ components such that $n_{1}$ of them each with its own weight $\omega_{i}$ and reliability $p_{1 i}$ and $n_{2}$ of them each with its own weight $\omega_{i}^{*}$ and reliability $p_{2 i}$. To operate the system, a certain capacity of components denoted by threshold $k$, should be provided.

The remainder of the paper is arranged as follows: in Section 2, for mentioned weighted- $k$-out-of- $n$ :G system, the description of system and its reliability evaluation is provided. The survival function and MTTF of this system are studied in Section 3. In Section 4, the Birnbaum reliability importance is investigated. Finally, the concluding remarks are given in Section 5.

\section{THE SYSTEM MODEL}

In this section, first some notations and the description of modelling main assumptions are provided for this general setup of weighted$k$-out-of- $n$ :G system.

\subsection{Notations}

\begin{tabular}{ll}
\hline$n$ & Number of components in a system \\
$X_{i}$ & State of $i$-th component of the system: \\
& $X_{i}=1$ if component is functioning; $X_{i}=0$ if it is failed \\
$k$ & Minimum total weight(capacity) of all working components to operate system \\
$C$ & The set of all components \\
$C_{1}$ & First group of components \\
$C_{2}$ & Second group of components \\
$n_{1}$ & Number of components in $C_{1}$ \\
$n_{2}$ & Number of components in $C_{2}$ \\
$\omega_{i}$ & Weight of $i$-th component in $C_{1}$ \\
$\omega_{i}^{*}$ & Weight of $i$-th component in $C_{2}$ \\
$p_{1 i}$ & Reliability of $i$-th component in $C_{1}$ \\
$p_{2 i}$ & Reliability of $i$-th component in $C_{2}$ \\
\hline
\end{tabular}

\subsection{System Description}

Consider a system including $n$ independent and nonidentical components which are placed in two groups with respect to their duties and services. Each component has its own positive integer-valued weight. Let the state of $i$-th component $X_{i}$ be an independent binary random variable such that $p_{1 i}=P\left(X_{i}=1\right)$ if $i \in C_{1}$ with corresponding weight $\omega_{i}$ and $p_{2 i}=P\left(X_{i}=1\right)$ if $i \in C_{2}$ with corresponding weight $\omega_{i}^{*}$. According to the above representation, clearly $C=C_{1} \cup C_{2}, C_{1} \cap C_{2}=\varnothing,\left|C_{1}\right|=n_{1}$ and $\left|C_{2}\right|=n_{2}$. Assume that $\phi(X)$ denotes the structure function of the system where $\boldsymbol{X}=\left(X_{1}, \cdots, X_{n}\right)$ is the state vector of components. As mentioned, in this general setup of weighted- $k$-outof- $n$ :G system, if the total weight of all functioning components is above predefined threshold $k$, the system is supposed to work. So, the structure function of this system is defined by

$$
\phi(X)= \begin{cases}1 & \text { if } \sum_{i \in C_{1}} \omega_{i} X_{i}+\sum_{i \in C_{2}} \omega_{i}^{*} X_{i} \geq k, \\ 0 & \text { o.w. }\end{cases}
$$

Evidently, the reliability of system defined as probability that system is working, can be given by the probability

$$
R=P(\phi(X)=1)=P\left(\sum_{i \in C_{1}} \omega_{i} X_{i}+\sum_{i \in C_{2}} \omega_{i}^{*} X_{i} \geq k\right) .
$$




\subsection{Reliability Evaluation}

Now, taking the independent and nonidentical components into account with the reliability of $i$-th component $p_{1 i}$ if $i \in C_{1}$ with corresponding weight $\omega_{i}$ and $p_{2 i}$ if $i \in C_{2}$ with corresponding weight $\omega_{i}^{*}$, the reliability of system defined by (1) can be formulated as follows:

$$
R=\sum_{s=0}^{n_{1}} \sum_{u=0}^{n_{2}} \sum_{\substack{E_{s} \\\left(E_{s}, E_{u}\right) \in \mathbb{E}}} \sum_{E_{u}} \prod_{m=1}^{s} p_{1 l_{m}} \prod_{m=s+1}^{n_{1}}\left(1-p_{1 l_{m}}\right) \prod_{m=1}^{u} p_{2 l_{m}} \prod_{m=u+1}^{n_{2}}\left(1-p_{2 l_{m}}\right)
$$

where $s=\sum_{i \in C_{1}} X_{i}, u=\sum_{i \in C_{2}} X_{i}$, summation $E_{s}\left(E_{u}\right)$ extends over all combinations $l_{1}, \cdots, l_{s}\left(l_{1}, \cdots, l_{u}\right)$ of $\left\{1, \cdots, n_{1}\right\}\left(\left\{1, \cdots, n_{2}\right\}\right)$ and

$$
\mathbb{E}=\left\{\left(E_{s}, E_{u}\right): \sum_{m=1}^{s} \omega_{l_{m}} X_{l_{m}}+\sum_{m=1}^{u} \omega_{l_{m}}^{*} X_{l_{m}} \geq k\right\}
$$

In the following, illustrative results are presented to observe the value of $R$ for a weighted- $k$-out-of-5:G system with respect to the different values of $k, n_{1}$ and the component weights and reliabilities.

\section{Example 2.1.}

Consider a weighted- $k$-out-of- $5: \mathrm{G}$ system with two especial case $n_{1}=2$ and $n_{1}=3$. First case $n_{1}=2$, suppose that $\omega_{1}=3, \omega_{2}=1, \omega_{1}^{*}=$

\begin{tabular}{|c|c|c|c|c|c|c|}
\hline \multicolumn{3}{|c|}{$C_{1}$} & \multicolumn{4}{|c|}{$C_{2}$} \\
\hline$i$ & 1 & 2 & $i$ & 1 & 2 & 3 \\
\hline$p_{1 i}$ & 0.95 & 0.97 & $p_{2 i}$ & 0.90 & 0.95 & 0.99 \\
\hline
\end{tabular}
$1, \omega_{2}^{*}=2$ and $\omega_{3}^{*}=3$ with corresponding component reliabilities as

Then for $k=7$,

$$
\begin{aligned}
R= & {\left[p_{11}\left(1-p_{12}\right)\left(p_{21}\left(1-p_{22}\right) p_{23}+\left(1-p_{21}\right) p_{22} p_{23}+p_{21} p_{22} p_{23}\right)+\left(1-p_{11}\right) p_{12} p_{21} p_{22} p_{23}\right] } \\
& +\left[p_{11} p_{12}\left(\left(1-p_{21}\right)\left(1-p_{22}\right) p_{23}+p_{21} p_{22}\left(1-p_{23}\right)+p_{21}\left(1-p_{22}\right) p_{23}+\left(1-p_{21}\right) p_{22} p_{23}+p_{21} p_{22} p_{23}\right)\right],
\end{aligned}
$$

\begin{tabular}{|c|c|c|c|c|c|c|}
\hline \multicolumn{4}{|c|}{$C_{1}$} & \multicolumn{3}{|c|}{$C_{2}$} \\
\hline$i$ & 1 & 2 & 3 & $i$ & 1 & 2 \\
\hline$p_{1 i}$ & 0.95 & 0.97 & 0.85 & $p_{2 i}$ & 0.90 & 0.95 \\
\hline
\end{tabular}

and for $k=9$,

$$
\left.R=\left[p_{11}\left(1-p_{12}\right) p_{21} p_{22} p_{23}\right)\right]+\left[p_{11} p_{12}\left(\left(1-p_{21}\right) p_{22} p_{23}+p_{21} p_{22} p_{23}\right)\right]
$$

Now case $n_{1}=3$, let $\omega_{1}=3, \omega_{2}=1, \omega_{3}=2, \omega_{1}^{*}=1$ and $\omega_{2}^{*}=2$ with corresponding component reliabilities as

The calculation of $R$ for $k=7$ and $k=9$ is similar.

In Table 1 , the reliability of the weighted- $k$-out-of- 5:G system is computed with respect to the different values of $k$, $n_{1}$ and the weight of components. As observed, the system reliability is sensitive to the values of $n_{1}$ which determines the number of components in each group and also, the values of threshold $k$ plays a significant role in system reliability.

Table $1 \mid$ Reliability of the weighted- $k$-out-of-5:G system.

\begin{tabular}{cccc}
\hline $\boldsymbol{n}$ & $\boldsymbol{n}_{\mathbf{1}}$ & $\boldsymbol{k}$ & $\boldsymbol{R}$ \\
\hline 5 & 2 & 7 & 0.9893 \\
& & 9 & 0.8910 \\
& 3 & 7 & 0.9210 \\
& & 9 & 0.6697 \\
\hline
\end{tabular}


Proposition 2.1. As an especial case, consider a weighted- $k$-out-of- $n: G$ system including independent and identical components in each of two groups with the reliability of $i$-th component $p_{1}$ if $i \in C_{1}$ and $p_{2}$ if $i \in C_{2}$, but with different corresponding weight $\omega_{i}$ and $\omega_{i}^{*}$ respectively in each group. Then, the reliability given by (2) can be rewritten as

$$
R=\sum_{s=0}^{n_{1}} \sum_{\substack{n_{2} \\ n_{s}}} \sum_{\substack{E_{s} \\\left(E_{s}, E_{u}\right) \in \mathbb{E}}} p_{1}^{s}\left(1-p_{1}\right)^{n_{1}-s} p_{2}^{u}\left(1-p_{2}\right)^{n_{2}-u}
$$

where the notations are mentioned for Equation (2).

Remark 2.1. If all the weights $\omega_{i}$ and $\omega_{i}^{*}$ are respectively equal to $\omega$ and $\omega^{*}$ with identical components in each of two groups, the especial case of weighted- $k$-out-of- $n: G$ system containing two types of components presented by Eryilmaz and Sarikaya [10] is concluded.

\section{SURVIVAL ANALYSIS}

Let $T_{1}, \cdots, T_{n}$ represent the lifetimes of $n$ independent and nonidentical components. As representative of the performance of the weighted$k$-out-of- $n: G$ system, the total weight of the system at time $t(\geq 0)$ can be described by

$$
W_{n}(t)=\sum_{i \in C_{1}} \omega_{i} I\left(T_{i}>t\right)+\sum_{i \in C_{2}} \omega_{i}^{*} I\left(T_{i}>t\right),
$$

where $I\left(T_{i}>t\right)$ is indicator function (which is 1 if $T_{i}>t$ and 0 if $T_{i} \leq t$ ). Suppose that the survival function of $i$-th component is $\bar{F}_{i}(t)=P\left(T_{i}>t\right), \forall i \in C_{1}$ and $\bar{G}_{i}(t)=P\left(T_{i}>t\right), \forall i \in C_{2}$, then the mean weight of the system at time $t$ is obtained as

$$
\begin{aligned}
E\left[W_{n}(t)\right] & =\sum_{i \in C_{1}} \omega_{i} P\left(T_{i}>t\right)+\sum_{i \in C_{2}} \omega_{i}^{*} P\left(T_{i}>t\right) \\
& =\sum_{i=1}^{n_{1}} \omega_{i} \bar{F}_{i}(t)+\sum_{i=1}^{n_{2}} \omega_{i}^{*} \bar{G}_{i}(t) .
\end{aligned}
$$

Now, considering $T$ as the lifetime of the system, then it is defined as

$$
T=\inf \left\{t: W_{n}(t)<k\right\}
$$

So, by substituting $p_{1 l_{m}}$ and $p_{2 l_{m}}$ in Equation (2) by $\bar{F}_{l_{m}}(t)$ and $\bar{G}_{l_{m}}(t)$, respectively, the survival function defined as probability that system is working at time $t$ for weighted- $k$-out-of- $n: G$ system with two type nonidentical components can be established as follows:

$$
S(t)=P(T>t)=\sum_{s=0}^{n_{1}} \sum_{u=0}^{n_{2}} \sum_{\substack{E_{s} \\\left(E_{s}, E_{u}\right) \in \mathbb{E}}} \sum_{E_{u}} \prod_{m=1}^{s} \bar{F}_{l_{m}}(t) \prod_{m=s+1}^{n_{1}} F_{l_{m}}(t) \prod_{m=1}^{u} \bar{G}_{l_{m}}(t) \prod_{m=u+1}^{n_{2}} G_{l_{m}}(t),
$$

where the notations are mentioned for Equation (2) in previous section.

Proposition 3.1. For the especial case mentioned in Proposition 2.1, substituting $\bar{F}(t)$ and $\bar{G}(t)$, respectively, the survival function (3) at time $t$ can be rewritten as

$$
S(t)=\sum_{s=0}^{n_{1}} \sum_{u=0}^{n_{2}} \sum_{\substack{E_{s} \\\left(E_{s}, E_{u}\right) \in \mathbb{E}}} \sum_{E_{u}} \bar{F}^{s}(t) F^{n_{1}-s}(t) \bar{G}^{u}(t) G^{n_{2}-u}(t)
$$

where the notations are mentioned for Equation (2) in previous section.

As one of the most important reliability characteristics, the MTTF of a system is defined as expected value of the lifetime of the system

$$
\operatorname{MTTF}=E(T)=\int_{0}^{\infty} P(T>t) d t .
$$

Hence, the MTTF of our proposed system is computed by

$$
\operatorname{MTTF}=\sum_{s=0}^{n_{1}} \sum_{u=0}^{n_{2}} \sum_{\substack{E_{s} \\\left(E_{s}, E_{u}\right) \in \mathbb{E}}} \sum_{E_{u}} \int_{0}^{\infty} \prod_{m=1}^{s} \bar{F}_{l_{m}}(t) \prod_{m=s+1}^{n_{1}} F_{l_{m}}(t) \prod_{m=1}^{u} \bar{G}_{l_{m}}(t) \prod_{m=u+1}^{n_{2}} G_{l_{m}}(t) d t .
$$




\section{Example 3.1.}

Considering the weighted- $k$-out-of- 5:G system mentioned in Example 2.1, let the survival function of $i$-th component for each classes is $\bar{F}_{i}(t)=e^{-\lambda_{i} t}$ and $\bar{G}_{i}(t)=e^{-\mu_{i} t}$, respectively. For first case $n_{1}=2$, suppose that $\omega_{1}=3, \omega_{2}=1, \omega_{1}^{*}=1, \omega_{2}^{*}=2$ and $\omega_{3}^{*}=3$ with corresponding component survival function $\lambda=(0.2,0.3)$ and $\mu=(0.6,0.1,0.4)$. For second case $n_{2}=3$, suppose that $\omega_{1}=3, \omega_{2}=$ $1, \omega_{3}=1, \omega_{1}^{*}=1$ and $\omega_{2}^{*}=2$ with corresponding component survival function $\lambda=(0.2,0.6,0.3)$ and $\mu=(0.1,0.4)$.

From Figure 1, the graphs of survival function with respect to different values of $k$ indicate that survival of system depends on the combination of the survival function and the weight of components in each of classes. Obviously, survival function is sensitive to the values of $n_{1}$ which determines the number of components in each group and also, the values of threshold $k$ plays a significant role in system reliability.

As well, MTTF is

$$
\begin{aligned}
\text { MTTF } & =\sum_{s=0}^{n_{1}} \sum_{u=0}^{n_{2}} \sum_{E_{s}} \sum_{E_{u}} \int_{0}^{\infty} \prod_{m=1}^{s} e^{-\lambda_{l_{m}} t} \prod_{m=s+1}^{n_{1}}\left(1-e^{-\lambda_{l_{m}} t}\right) \prod_{m=1}^{u} e^{-\mu_{l_{m}} t} \prod_{m=u+1}^{n_{2}}\left(1-e^{-\mu_{l_{m}} t}\right) d t, \\
& =\sum_{s=0}^{n_{1}} \sum_{u=0}^{n_{2}} \sum_{\substack{\left.E_{s}, E_{u}\right) \in \mathbb{E} \\
\left(E_{s}, E_{u}\right) \in \mathbb{E}}} \sum_{E_{u}} \int_{0}^{\infty} e^{-t\left(\sum_{m=1}^{s} \lambda_{l_{m}}+\sum_{m=1}^{u} \mu_{l_{m}}\right)} \prod_{m=s+1}^{n_{1}}\left(1-e^{-\lambda_{l_{m}} t}\right) \prod_{m=u+1}^{n_{2}}\left(1-e^{-\mu_{l_{m}} t}\right) d t .
\end{aligned}
$$

In Table 2, the MTTF of the weighted- $k$-out-of- 5:G system is computed with respect to the different values of $k$, $n_{1}$ and the weight of components. As observed, MTTF is sensitive to the values of $n_{1}$ which determines the number of components in each group and also, the values of threshold $k$ plays a significant role in system reliability.

\section{COMPONENT IMPORTANCE}

Considering the important role of ranking the components according to their importance measure in system reliability, the Birnbaum reliability importance is obtained in a weighted- $k$-out-of- $n: G$ system with two type nonidentical components. Birnbaum [12] introduced the importance of the $i$-th component in a coherent system. If the event $S$ shows that the system works, then Birnbaum reliability importance of the $i$-th component is defined by

$$
I_{i}=P\left(S \mid X_{i}=1\right)-P\left(S \mid X_{i}=0\right)
$$
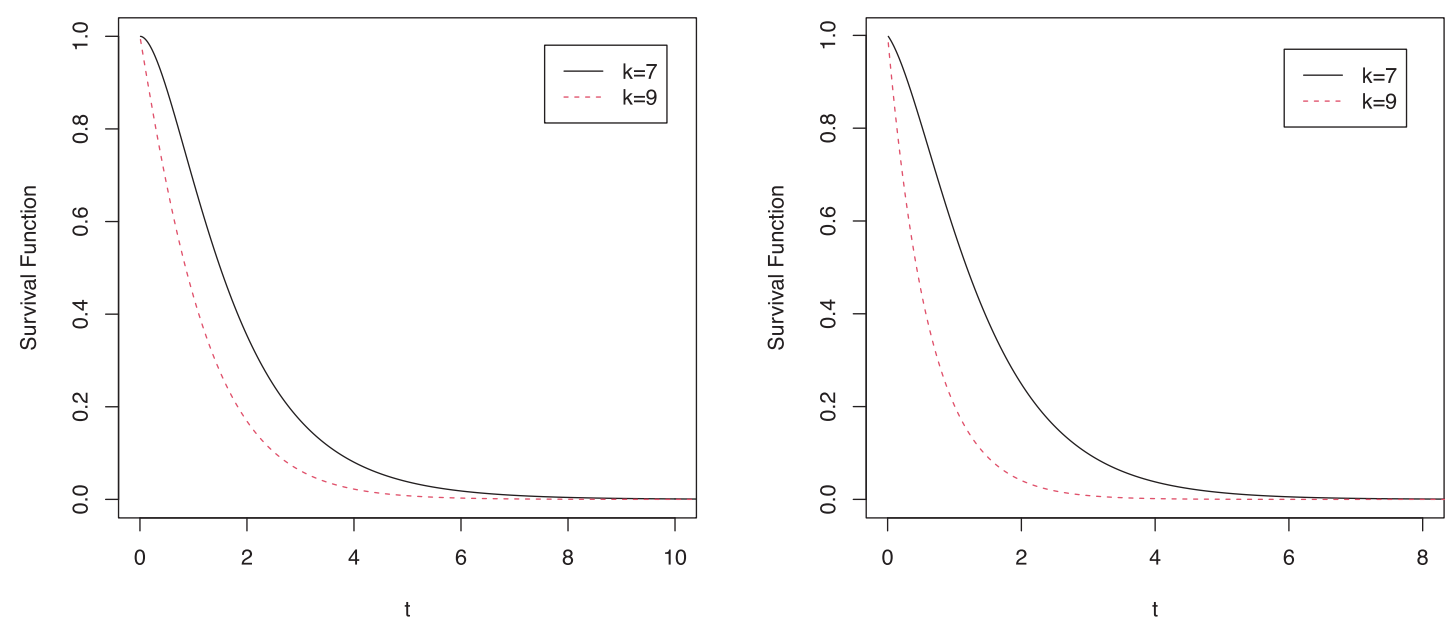

Figure 1 The survival function with respect to different values of (right side).

Table 2 Mean time to failure (MTTF) of the weighted- $k$-out-of-5:G system.

\begin{tabular}{llll}
\hline $\boldsymbol{n}$ & $\boldsymbol{n}_{\mathbf{1}}$ & $\boldsymbol{k}$ & MTTF \\
\hline 5 & 2 & 7 & 1.8597 \\
& & 9 & 1.1442 \\
& 3 & 7 & 1.4637 \\
& & 9 & 0.6250 \\
\hline
\end{tabular}


In this setup of weighted- $k$-out-of- $n: G$ system, the Birnbaum reliability importance of the $i$-th component is rewritten as follows:

For $i \in C_{1}$,

$$
P\left(S \mid X_{i}=1\right)=\sum_{s=1}^{n_{1}} \sum_{u=0}^{n_{2}} \sum_{\substack{E_{s \mid i} \\\left(E_{s i}, E_{u}\right) \in \mathbb{E}}} \prod_{E_{u}}^{s-1} p_{m=1} \prod_{m} \prod_{m=s+1}^{n_{1}}\left(1-p_{1 l_{m}}\right) \prod_{m=1}^{u} p_{2 l_{m}} \prod_{m=u+1}^{n_{2}}\left(1-p_{2 l_{m}}\right),
$$

and

$$
P\left(S \mid X_{i}=0\right)=\sum_{s=0}^{n_{1}-1} \sum_{u=0}^{n_{2}} \sum_{\substack{E_{s \backslash i} \\\left(E_{s \backslash}, E_{u}\right) \in \mathbb{E}}} \prod_{m=1}^{s} p_{1 l_{m}} \prod_{m=s+1}^{n_{1}-1}\left(1-p_{1 l_{m}}\right) \prod_{m=1}^{u} p_{2 l_{m}} \prod_{m=u+1}^{n_{2}}\left(1-p_{2 l_{m}}\right),
$$

where summation $E_{s \backslash i}$ extends over all combinations $l_{1}, \cdots, l_{s}$ of $\left\{1, \cdots, i-1, i+1, \cdots, n_{1}\right\}$, denoted by $\left\{1, \cdots, n_{1}\right\} \backslash\{i\}$.

For $i \in C_{2}$,

$$
P\left(S \mid X_{i}=1\right)=\sum_{s=0}^{n_{1}} \sum_{u=1}^{n_{2}} \sum_{\substack{E_{s} \\\left(E_{s}, E_{u \backslash i}\right) \in \mathbb{E}}} \sum_{E_{u \backslash}} \prod_{m=1}^{s} p_{1 l_{m}} \prod_{m=s+1}^{n_{1}}\left(1-p_{1 l_{m}}\right) \prod_{m=1}^{u-1} p_{2 l_{m}} \prod_{m=u+1}^{n_{2}}\left(1-p_{2 l_{m}}\right),
$$

and

$$
P\left(S \mid X_{i}=0\right)=\sum_{s=0}^{n_{1}} \sum_{u=0}^{n_{2}-1} \sum_{\substack{E_{s} \\\left(E_{s}, E_{u \backslash i}\right) \in \mathbb{E}}} \prod_{m=1}^{s} p_{1 l_{m}} \prod_{m=s+1}^{n_{1}}\left(1-p_{1 l_{m}}\right) \prod_{m=1}^{u} p_{2 l_{m}} \prod_{m=u+1}^{n_{2}-1}\left(1-p_{2 l_{m}}\right),
$$

where summation $E_{u \wedge i}$ extends over all combinations $l_{1}, \cdots, l_{u}$ of $\left\{1, \cdots, n_{2}\right\} \backslash\{i\}$. Let $I_{1}\left(I_{2}\right)$ denote the importance of the components in $C_{1}\left(C_{2}\right)$ that equals the difference between Equations (5) and (6) (Equations (7) and (8)). Next example presents the value of Birnbaum reliability importance for a weighted- $k$-out-of- 5:G system with respect to the values of $k, n_{1}$, and the component weights and reliabilities.

\section{Example 4.1.}

Consider a weighted- $k$-out-of- 5:G system with the especial case $n_{1}=3$ and $k=5$. Suppose that $\omega_{1}=3, \omega_{2}=1, \omega_{3}=2, \omega_{1}^{*}=1$ and $\omega_{2}^{*}=2$ with corresponding component reliabilities $p_{1}=\left(p_{11}, p_{12}, p_{13}\right)$ and $p_{2}=\left(p_{21}, p_{22}\right)$.

For instance, if $i=3 \in C_{1}$,

$$
\begin{aligned}
P\left(S \mid X_{i}=1\right)= & {\left[\left(1-p_{11}\right)\left(1-p_{12}\right) p_{21} p_{22}\right]+\left[p_{11}\left(1-p_{12}\right)\left(\left(1-p_{21}\right)\left(1-p_{22}\right)+p_{21}\left(1-p_{22}\right)+\left(1-p_{21}\right) p_{22}+p_{21} p_{22}\right)\right] } \\
& +\left[\left(1-p_{11}\right) p_{12}\left(\left(1-p_{21}\right) p_{22}+p_{21} p_{22}\right)\right]+\left[p_{11} p_{12}\left(\left(1-p_{21}\right)\left(1-p_{22}\right)+p_{21}\left(1-p_{22}\right)+\left(1-p_{21}\right) p_{22}+p_{21} p_{22}\right)\right],
\end{aligned}
$$

and

$$
\begin{aligned}
P\left(S \mid X_{i}=0\right)= & {\left[p_{11}\left(1-p_{12}\right)\left(\left(1-p_{21}\right) p_{22}+p_{21} p_{22}\right)\right] } \\
& +\left[p_{11} p_{12}\left(p_{21}\left(1-p_{22}\right)+\left(1-p_{21}\right) p_{22}+p_{21} p_{22}\right)\right] .
\end{aligned}
$$

In Table 3, the Birnbaum reliability importance of the weighted- $k$-out-of- 5:G system is computed with respect to the different vectors of reliability of the components in both group $C_{1}$ and $C_{2}$. As observed, the value of $I_{1}$ and $I_{2}$ depends on the combination of the weight and the reliability of the components.

\section{CONCLUSION}

In many situations, the components contribute differently to the capacity of the system which the weighted- $k$-out-of- $n$ :G system are used to deal with. A system including $n$ components with their different positive integer weights that it works if and only if the total weight of working components is above a given threshold $k$. In most of the studies in the literature, the weighted- $k$-out-of- $n$ :G systems have been studied with identical components.

In this paper, we introduce a special case of weighted- $k$-out-of- $n$ :G system formed from two types of nonidentical components with different weights in which one group consists of $n_{1}$ components each with its own positive integer-valued weight $\omega_{i}$ and reliability $p_{1 i}$, 
Table 3 Birnbaum reliability importance of the weighted- $k$-out-of-5:G system for $n_{1}=3$ and $k=5$ with respect to reliability of the components.

\begin{tabular}{ccccccc}
\hline $\boldsymbol{P}_{\mathbf{1}}$ & $\boldsymbol{P}_{\mathbf{2}}$ & $\boldsymbol{i}$ & $\boldsymbol{\omega}_{\boldsymbol{i}}$ & $\boldsymbol{\omega}_{\boldsymbol{i}}^{*}$ & $\boldsymbol{I}_{\mathbf{1}}$ & $\boldsymbol{I}_{\mathbf{2}}$ \\
\hline$(0.95,0.97,0.85)$ & $(0.90,0.95)$ & 1 & 3 & 1 & 0.1940 & 0.0102 \\
& & 2 & 1 & 2 & 0.0105 & 0.0605 \\
$(0.80,0.90,0.95)$ & $(0.90,0.85)$ & 1 & 3 & 1 & 0.1992 & 0.0472 \\
& & 2 & 1 & 2 & 0.0516 & 0.1957 \\
$(0.80,0.90,0.85)$ & $(0.95,0.97)$ & 1 & 3 & 1 & 0.1790 & 0.0243 \\
& & 2 & 1 & 2 & 0.0117 & 0.1866 \\
$(0.70,0.75,0.65)$ & $(0.85,0.65)$ & 1 & 3 & 1 & 0.5490 & 0.1472 \\
& & 2 & 1 & 2 & 0.0919 & 0.2765 \\
& & 3 & 2 & - & 0.2765 & - \\
\hline
\end{tabular}

while the other group consists of $n_{2}$ components each with its own positive integer-valued weight $\omega_{j}^{*}$ and reliability $p_{2 j}$, $\left(n=n_{1}+n_{2}\right)$. The components are categorized into two groups with respect to their duties and services with different capacity. If the total weights of the functioning components exceeds a prespecified threshold $k$, the system is supposed to work. The reliability and component importance of this system are studied. The survival function and MTTF are presented. This setup of weighted- $k$-out-of- $n$ :G system containing two types of components might be useful in practice. As result, the system reliability, survival function, MTTF and component importance of this system are sensitive to the values of $n_{1}$ which determines the number of components in each group and also, the values of threshold $k$ plays a significant role in system reliability.

\section{CONFLICTS OF INTEREST}

There is no conflict of interest in this article. Mrs Meshkat is the phd student under supervision of Prof. Eisa Mahmoudi and this work is related to her thesis.

\section{AUTHORS' CONTRIBUTIONS}

RahmatSadat Meshkat wrote the initial draft of the paper and did the analysis. Eisa Mahmoudi helped in analyzing the finding and supervised overall work.

\section{Funding Statement}

The work is sponsored by the Yazd University, Iran. We have received no Funding for this paper.

\section{ACKNOWLEDGMENTS}

The authors would like to sincerely thank the Editor-in-Chief, Associate Editor, and referees for carefully reading the paper and for their useful comments. The authors are also indebted to Yazd University for supporting this research.

\section{REFERENCES}

1. J.S. Wu, R.J. Chen, IEEE Trans. Reliab. 43 (1994), 327-328.

2. Y. Chen, Q. Yang, IEEE Trans. Reliab. 54 (2005), 431-440.

3. F.J. Samaniego, M. Shaked, Stat. Probab. Lett. 78 (2008), 815-823.

4. W. Li, M.J. Zuo, Reliab. Eng. Syst. Saf. 93 (2008), 160-167.

5. J. Navarro, F.J. Samaniego, N. Balakrishnan, J. Appl. Probab. 48 (2011), 856-867.

6. S. Eryilmaz, Reliab. Eng. Syst. Saf. 109 (2013), 41-44.

7. S. Eryilmaz, A.R. Bozbulut, Comput. Ind. Eng. 72 (2014), 255-260.

8. R.A. Rahmani, M. Izadi, B.E. Khaledi, J. Comput. Appl. Math. 296 (2016), 1-9.

9. R.S. Meshkat, E. Mahmoudi, J. Comput. Appl. Math. 326 (2017), 273-283.

10. S. Eryilmaz, K. Sarikaya, Proc. Inst. Mech. Eng. O J. Risk Reliab. 228 (2014), 265-271.

11. S. Eryilmaz, J. Comput. Appl. Math. 346 (2019), 591-598.

12. Z.W. Birnbaum, in: P.R. Krishnaiah (Ed.), Multivariate Analysis II, Academic Press, New York, 1969, pp. 581-592. 